\title{
ACO-Based Power Allocation for Throughput Maximization in the Downlink 5G NOMA Systems
}

\author{
Osama Abuajwa ${ }^{1}$, Chee Keong Tan ${ }^{2}$, Yin Hoe $\mathrm{Ng}^{1}$ and Ching Kwang Lee ${ }^{1}$ \\ ${ }^{\text {I}}$ Faculty of Engineering; Multimedia University, Cyberjaya, Malaysia. \\ ${ }^{2}$ School of Information Technology; Monash University, Subang Jaya, Malaysia.
}

ORCID: 0000-0002-0971-5657 (Osama Abuajwa)

\begin{abstract}
Non-Orthogonal Multiple Access (NOMA) is a radio access technology that can increase the spectral efficiency of $5 \mathrm{G}$ networks. In a NOMA system, the power allocation is a combinatorial optimization problem since multiple users are multiplexed on the same radio resources. The power transmitted for each subchannel and the multiplexed users can be allocated by using metaheuristic methods to improve the system throughput. In this paper, ant colony optimization (ACO) is proposed to optimize the transmit power to maximize the overall throughput in the downlink of 5G NOMA systems. The ACO is one of the swarm intelligence algorithms that ensures that the optimal solution can be found via a number of iterations with specific values of parameters. The obtained results demonstrate that the proposed power allocation scheme using ACO performs better in terms of the system throughput by up to $35 \%$.
\end{abstract}

Keywords: Ant Colony Optimization (ACO), Power Allocation (PA), Non-Orthogonal Multiple Access (NOMA), Orthogonal Multiple Access (OMA), Successive Interference Cancellation (SIC).

\section{INTRODUCTION}

Modern 5G wireless networks have been experiencing an increasingly high demand for massive mobile connectivity which lead to spectral scarcity. Although the traditional orthogonal multiple access (OMA) can serve multiple users simultaneously by using orthogonal resources, the availability of the orthogonal resources is limiting the number of users. Furthermore, 5G networks targeted a high system capacity, while OMA is not suitable to satisfy this demand [1].

On the other hand, non-orthogonal multiple access (NOMA) allows multiple users to share resources simultaneously either in time, frequency, or code domains. The numbers of available non-orthogonal resources and scheduling granularity enable NOMA to support more devices or users than OMA [1].The only drawback of this approach is the generation of signal interference due to the sharing of the resources. Hence, signal interference cancellation (SIC) techniques have been adopted at one of the receiving ends in the NOMA system.

Various optimization techniques have been widely proposed to solve resource allocation problems for NOMA-based system. Zhiguo Ding has proposed the NOMA system in cooperating with multi-input multi-output (MIMO) technologies for the downlink scenario with two users [2]. He reported that massive throughput could be achieved by designating various power levels for multiple users in comparison to conventional OMA.

The work in [3] has proposed similar power allocation techniques for both downlink single-input-single-output (SISO) and MIMO-based NOMA scenarios. Both [2] and [3] have demonstrated that power allocation (PA) is capable of improving the system throughput performance significantly. The authors in [4] have applied an iterative sub-optimal distribution of power to two users multiplexed over a sub-band and across different sub-bands to achieve maximum throughput. This work shows that the weighted sum rate of orthogonal frequency-division multiple-access (OFDMA) based on NOMA system can be maximized by using the difference of convex programming (DC) approach.

Another investigation conducted by [5] has illustrated that an optimal solution for power allocation was obtained using the Lagrange multiplier technique for the two-users' scenario for the maximization of the sum rate in a NOMA system. The proposed algorithm achieved a better sum-rate performance than the OFDMA. The authors then extended the work to multiple users [5]. However, excessive complexity resulted from this optimal solution that needs to be addressed.

A hybrid method that combines user scheduling, resource allocation, and power control schemes is presented in [6] to maximize the total throughput of a NOMA system. The optimal power in a closed-form is derived in [6] for a downlink NOMA system considering perfect and imperfect of the channel state information (CSI) to maximize the system throughput. Additionally, the optimal bandwidth allocation closed-form is achieved when the logarithmic utility function is applied to satisfy the quality of service (QoS) for each user. Moreover, the Hungarian algorithm proposed in [6] to reduce the complexity of the hybrid resource allocation problem by performing an effective user pairing.

The iterative water-filling approach provided in [7] operates based on power allocation directly to the single-user MIMO (SU-MIMO) to maximize the system throughput by using the low-complexity proportional fairness $(\mathrm{PF})$ scheduler. The PF 
scheduler is applied to make users multiplexed over the transmit antenna and individual sub-band. Higher throughput gain is recorded at the far end by water filling (WF) at the expense of the user's suffering less throughput to those users closer to the base station [7]. The WF principle reported in [8] allocates power to the multiplexed users directly without considering sub-band allocation, which leads to the degradation of the achievable system throughput. The work in [9] investigated the NOMA performance with (SU-MIMO) in multi-cell system level for power allocation and multi-user pairing with practical assumption using adaptive modulation and coding (AMC) and feedback channel quality indicator (CQI).

In addition, the proposed NOMA system approach for 30 users achieved a 51\% throughput gain more than OMA [9] that gain about 39\%.Moreover, the monotonic optimization approach proposed in [10] has provided a solution to address the combined non-convex optimization issues of power and subcarrier allocation to maximize the weighted system throughput. The monotonic optimization approach was used for the optimal resource allocation, which is adopted as the performance benchmark even though it has high complexity. The resource allocation problem has to include subchannel allocation in addition to power allocation which increases the complexity in the NOMA system, as presented in [10].

The genetic approach (GA) is one of the meta-heuristic algorithms applied in [11] to cluster multiple users for NOMA downlink system where the obtained optimal transmit power levels are assigned to each user group. The GA in [11] is applied jointly by combining power allocation and user grouping based on resource allocation to achieve the maximum geometric mean of the user throughput.

Ant Colony Optimization (ACO) is another meta-heuristic algorithm employed to solve combinatorial optimization problems. The ACO algorithm is a more efficient approach of the ant colony system that has been evolved from the first algorithm Ant System (AS) [12].The multi-user resource allocation required a specific model for the application of ACO to ensure the combinatorial optimization problem is feasible for the OFDMA system [13].

The two essential attributes in ACO are the best individual selection, which is equivalent to using memory and randomness that can improve the search efficiency. This attribute in ACO assists in enhancing individual selection and effectively considers the most suitable solution. These are essential features in preventing the solution of trapping within local optimum [14] [15]. The algorithm can converge and guarantees an optimal solution. The optimization of power allocation is classified under Nondeterministic Polynomial (NP) NP-hard problem[11].

In the literature review, the majority of the found work focused on resource allocation to improve the performance of the throughput for the NOMA system. Since the resource allocation is defined as non-convex [4], a method is required to transform the non-convex into a convex problem that can achieve an effective solution. However, there are no tractable solutions that can be guaranteed for a non-convex optimization problem while converting the problem into sub-problem produces a suboptimal solution. ACO algorithm works iteratively to obtain the optimal solution, which can be more realistic and reliable in solving a non-convex optimization problem.

In NOMA system-based power domain multiplexing, multiple users are sharing the same radio resources where it requires a feasible power distribution for the users multiplexed on the same subchannel. Hitherto, no literature has been reported in applying ACO to the issues related to power allocation in NOMA system. The ACO algorithm is adopted to solve the problem of the power allocation in the NOMA system. Moreover, ACO is developed to optimize the transmit power for each subchannel, where the search solution is designed to form a graph of nodes of power levels, which are the set of power transmitted from Bs to the available subchannels. In the search, each subchannel explores the suitable power level that can maximize the system throughput. Artificial ants search for the short path by moving between the nodes looking for the power levels for each subchannel that maximize the system throughput. This search is performed iteratively through updating the pheromone on each edge and the path between edges to allow other ants to follow the solution giving the highest system throughput. The best solution will be the node with the most more visits and the shortest path connecting the nest and source of the food. The proposed ACO algorithm would have apparent advantages because of the search property that giving each subchannel probability to find the optimal solution quickly.

In this work, we propose a novel subchannel power allocation scheme for the downlink of the 5G NOMA system using ACO. The algorithm's search solution space is designed using the nodes of transmit power levels allocated to nodes of the subchannel hosting the multiplexed users. The remainder of the paper is organized as follows. The NOMA system model is presented in Section II as well as the mathematical formulation of the transmit power optimization problem for the downlink of the 5G NOMA system. Section III provides full detail of the proposed ACO based power allocation scheme. In Section IV, a comparative performance evaluation between the proposed scheme and the other available existing techniques is investigated and discussed. Finally, conclusions are drawn in Section V.

\section{II.SYSTEM MODEL AND PROBLEM FORMULATION}

Fig.1 depicts the SISO NOMA downlink system with two users multiplexed on the same subchannel. The base station (BS) transmits superimposed messages in a particular subcarrier to the multiplexed users using different powers based on channel 
gains difference of the user equipment (UEs).

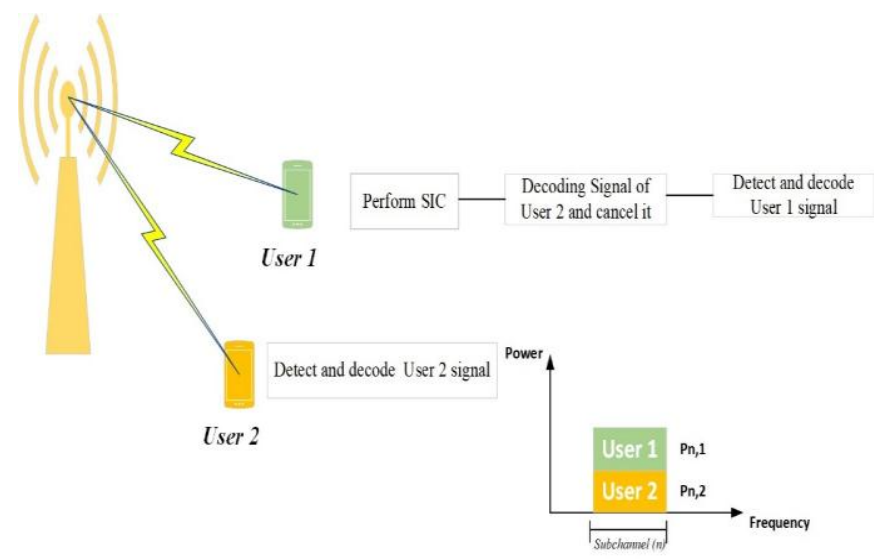

Fig.1.Basic NOMA system with two users multiplexed on the same subchannel with the assumption that $G_{1, n}>G_{2, n}$

At the receiver with higher channel gain, SIC is utilized to cancel the interference between the multiplexed users on a particular subcarrier so that the intended signal can be successfully retrieved. The signal $X_{i, n}$ is transmitted from BS to user $\boldsymbol{i}$ where $(i \in\{1,2, \ldots ., U\})$ over the $n t h$ subcarrier given as $\left(n \in\left\{1,2, \ldots . ., N_{s c}\right\}\right)$. In general, the received signal for user $\boldsymbol{u}$ in a NOMA system is given as follow:

$$
Y_{u}=\sum_{i=1}^{U} \sqrt{P_{i, n}} G_{i, n} X_{i, n}+N_{i}
$$

where $N_{i}$ is the additive white Gaussian noise (AWGN) and $G_{i, n}$ is the channel gain between the $i t h$ user and BS on the $n t h$ subcarrier respectively. The number of subcarriers is denoted as $N_{s c}$,which is used to determine the subchannel bandwidth from the available aggregate bandwidth $W_{T}$. The subcarrier bandwidth is described as $B_{s c}=W_{T} / N_{s c}$.

In a NOMA system, every two users share the same subchannel, and the instantaneous channel state information (CSI) is updated for each user. The $P_{u, n}$ is the power level allocated for each multiplexed user on a particular subchannel and the maximum transmit power is $P_{\max }$. Moreover, in the two-user NOMA system scenario, $P_{1, n}$ and $P_{2, n}$ are the transmit powers for each user multiplexed on the same subchannel, respectively. While the $\gamma_{1, n}, \gamma_{2, n}$ are the signal-to-interference plus noise ratio (SINR) for the two users who share the same subchannel ,respectively. The SINR of each multiplexed user is expressed as follows [5].

$$
\gamma_{1, n}=\frac{P_{1, n}\left|G_{1, n}\right|^{2}}{P_{2, n}\left|G_{1, n}\right|^{2}+B_{s c} N 。}
$$

$$
\gamma_{2, n}=\frac{\boldsymbol{P}_{2, n}\left|G_{2, n}\right|^{2}}{\boldsymbol{B}_{s c} N_{\circ}}
$$

where $N$ o represents the noise power spectral density. Referring to Fig.1, assuming User 1's channel condition experiencing relatively higher channel gain than User 2.The message of User 2 will be decoded by itself from the received signal, and any signal from User 1 will be treated as noise. Hence, it would be easier for User 1 to carry out SIC by cancelling the signal from User 2 and ensuring User 1 only received signal assigned to it. In the NOMA system with two user scenarios, the throughput for User 1 and User 2 are as follows:

$$
\begin{aligned}
& R_{U s e r 1}=B_{s c} \sum_{n=1}^{N_{s c}} \log _{2}\left(1+\gamma_{1, n}\right) \\
& R_{U s e r 2}=B_{s c} \sum_{n=1}^{N_{s c}} \log _{2}\left(1+\gamma_{2, n}\right)
\end{aligned}
$$

The total throughput for a NOMA system with two users can be written in (6) according to the concepts of Shannon's capacity formula. The throughput maximization of the two-user NOMA system is given with constraints in (7) and (8).

$$
\begin{gathered}
\operatorname{Max} R_{\text {sum }}=B_{s c} \sum_{n=1}^{N_{s c}} \log _{2}\left(1+\gamma_{u, n}\right)+B_{s c} \sum_{n=1}^{N_{s c}} \log _{2}\left(1+\gamma_{u, n}\right) \\
\text { Subject to } \quad c_{1}: \sum_{u=1}^{U} \sum_{n=1}^{N} P_{u, n} \leq P_{\max } \\
c_{2}: 0 \leq r_{\text {min }} \leq R_{\text {sum }}
\end{gathered}
$$

The condition $c_{1}$ in (7) guarantees that the total of subchannels and users transmit power is limited to the total BS power constraint and $c_{2}$ in (8) ensure the achievable throughput must be at least obtain $r_{\min }$ which is the minimum target rate .

\section{III.ANT COLONY OPTIMIZATION BASED POWER ALLOCATION}

ACO's concept originated from Macro Dorigo in 1997 to find the shortest path among the available routes to reach their destinations in solving the travelling salesman problem (TSP) [15]. ACO imitates the ant colony's behavior where the ant goes in searching for food source from its nest through the shortest path. The first ant moves randomly until it discovers the food source, it will then mark the route and the source by laying down a pheromone trail on its way back to the nest. Other ants will randomly follow and also create pheromone trails. The ants on the shortest path laying pheromone that creating higher pheromone concentration compared to different paths. Thus, making it more attractive to the succeeding ants to follow that path. In the longer path, pheromone evaporates eventually. Finally, the shortest path is identified. 
The prime objective in our work is to develop an ACO algorithm to yield an optimum power distribution solution to the subchannel, which provides maximum throughput for a NOMA-based $5 \mathrm{G}$ network. The development of our proposed ACO involves several steps, as illustrated in the flowchart presented in Fig.2.

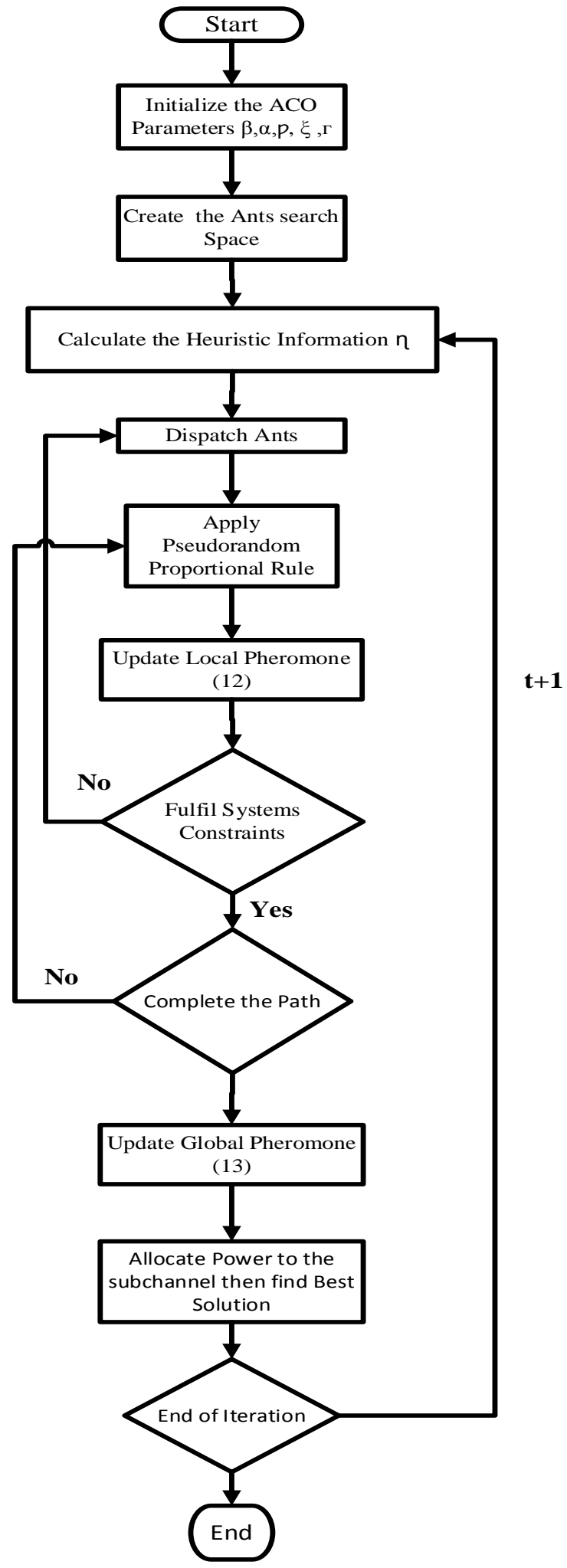

Fig.2. Flowchart of the proposed ACO based power allocation scheme for the downlink of 5G NOMA systems
In Fig.2, the first step is to initialize the parameters of the algorithm. It follows by constructing the search space, which consists of the power levels and subchannels nodes. The following step is the heuristic information evaluation, which provides the priori information that assists ants in choosing the next node. Thirdly, the state transition rule of ants that help in exploring or exploiting the offered solutions. The final step is to update local and global pheromone concentration continuously to achieve the optimal solution. These steps run based on iteration $t$ and the same process $t+1$ until the algorithm reaches the maximum number of iterations.

\section{III.I SEARCH SPACE}

Search space is created to emulate the ants moving from the nest to the source of the food to create potential solutions, as illustrated in Fig.3.In the ACO algorithm, the search space maintains a graphic of which nodes denote steps in ants' search for solutions for the problem. In our proposed algorithm, the search space is represented as $L \times N$ nodes where the total number of subchannels is $N$ and $L$ is the total number of power levels associated with a particular subchannel. For instance, the power level $P_{L, n}$ is emitted from the BS to subchannel $n$ that hosts the multiplexed users. Each node represents the pair of subchannel and power level. In our case, each subchannel assumed to accept only two multiplexed users.

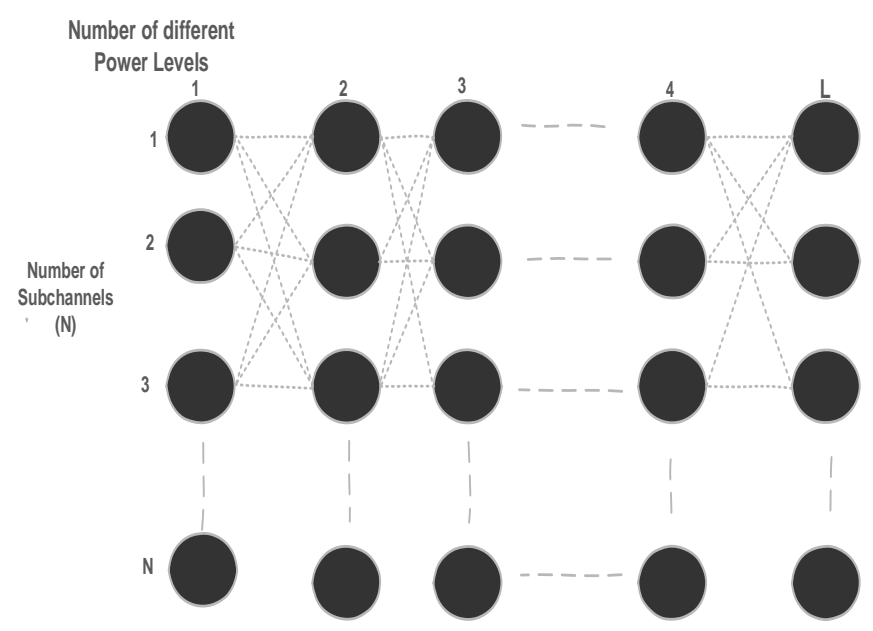

Fig.3. Search Space of the proposed ACO algorithm

The proposed algorithm of the ant colony optimization has to develop the solution search for the movement of ants between nodes to construct the optimal solution that maximizes system throughput performance. Ants will begin from the nest and shift between nodes of the $N$ column to another to allocate the power level on a particular subchannel for the mapped pair users until the ant reaches the food source. Furthermore, the power levels mainly represent the power transmitted from the Bs to the available subchannels. The total transmit power from the BS is divided randomly into different numbers of power levels as $P_{L}=\left[P_{L 1}, P_{L 2}, \ldots, P_{L}\right]$ subject to the constraints given in (7) 
and (8).The power levels are closer to the continuous transmit power that representing the subchannel power in the fitness function.

The transmit power levels are assigned by not exceeding the entire set of values for the search power levels. This process of power level assignment to subchannel nodes will continue until maximum throughput is obtained for that particular node. Moreover, that power level is recorded as local maximum, and then it will be used to reach the global solution while ants are continuously moving between the nodes as well as assigning the optimal power levels for the entire subchannels. The ACO's feasible solution can be satisfied when the heuristic information constantly changes node sizes during the ant's movement. The fitness function used in our proposed algorithm only considers power distribution and power ratio for the multiplexed users in a subchannel. The fitness function to maximize total throughput is formulated below.

$$
f\left(\delta, P_{n}\right)=B_{s c} \sum_{n=1}^{N_{s c}} \log _{2}\left(1+\delta P_{n} \gamma_{u, n}\right)+B_{s c} \sum_{n=1}^{N_{s c}} \log _{2}\left(1+\frac{(1-\delta) P_{n} \gamma_{u, n}}{1+\delta P_{n} \gamma_{u, n}}\right)
$$

where $\delta$ is the power distributed amongst the multiplexed users on the same sub-channel randomly ranging between 0 and 1 denoted as $0 \leq \delta \leq 1$.

\section{III.II THE HEURISTIC INFORMATION EVALUATION}

In general, heuristic evaluation is based on trial and error searching for the best optimal solution through evolution. The quality of each node is evaluated heuristically. This information will assist the succeeding ants to determine the next course of action. The process of dispatching ants will continue until all the available nodes are updated. This is similar to the visibility step in the travelling salesman problem (TSP). The node with higher information shall be selected for further estimation [12].The heuristic information is the throughput for each subchannel with a particular allocated power level where each subchannel multiplexed only two users.

The power levels, $P_{l, n}$, is the selected optimized value of a particular subchannel of the two users that yields maximum throughput. In general, the estimated throughput on a particular node is $r_{l, n}$ which is normalized and defined $\eta_{l, n}$ as the value of the heuristic information as given follow:

$$
\eta_{l, n}=\frac{r_{l, n}}{\sum_{n=1}^{N} R_{l, n}}
$$

The normalized function of the node with the power level $\left(P_{l, n}\right)$ is the estimated throughput on that particular subchannel divided by the sum throughput on the rest of nodes [15].

\section{III.III ANTS' STATE TRANSITION RULE}

There is a movement of ant $k$ between nodes to discover the optimal power solution at individual iteration for the modelled problem. The strategy assumes that ant $k$ is randomly lies on column $n$ for the current subchannel then uses the probability to choose the power level. The pseudo-random proportional rule states that the ants are moving in the search space to select the power level based on the trade-off between the exploitation and biased exploration [12], as shown below.

$$
P_{1, \mathrm{n}}=\left\{\begin{array}{c}
\arg \max _{P_{L} \in[1,2, \ldots L]}\left(\left[\tau_{l, n}\right]^{\alpha}\left[\eta_{l, n}\right]^{\beta}\right), \text { if } q \leq q_{o},(\text { exploitation }) \\
\left.P_{1, \mathrm{n}}=\frac{\left[\tau_{l, n}\right]^{\alpha}\left[\eta_{l, n}\right]^{\beta}}{\sum_{\mathrm{k}=1}^{N}\left[\tau_{\mathrm{k}, \mathrm{n}}\right]^{\alpha}\left[\eta_{\mathrm{k}, \mathrm{n}}\right]^{\beta}}, \text { Else , (biased exploration }\right)
\end{array}\right.
$$

The random number $q$ in equation (11) is uniformly distributed $[0,1]$ and $q_{0}$ is a parameter satisfying $0 \leq q_{0} \leq 1$.The settings for the regulation of the pheromone influence and heuristic information influence $\left(\eta_{l, n}\right)$ are $\alpha$ and $\beta$ respectively. $P_{L}$ is the set of available power levels for ant $k$ to be allocated to subchannel $n$ which will be updated after each allocation process. The selection of the current best power level is on the basis of the exploitation for every time ant $k$ on subchannel node to choose the power level, if $q \leq q_{0}$ according to (11-a). This exploitation process ensures the current power level is allocated to that particular subchannel. Further, ants intend to choose the node with greater pheromone concentration and higher heuristic value.Meanwhile, the choice of the power level is based on the biased exploration, represented as $\mathcal{P}_{l, n}$. This is the random variable that will have to be chosen by the use of random proportional rule shown in (11-b), providing the ant's probability distribution to choose the node of candidate's power level for that subchannel node [12].

The set of remaining power levels nodes are the candidate's solutions for the ant $k$ on the current node to be visited to ensure that the power level set can give a feasible solution. The ant stops moving anymore and immediately eliminated, if the total power allocated to the subchannels exceeded the BS power constraints. In addition, a new ant will be dispatched again to continue allocating power levels to the subchannels until the path between the nest and food source is finished.

\section{III.IV PHEROMONE UPDATING}

This step addresses the ant's movement based on the pheromone evaporation rate and deposited concentration. An updated local pheromone concentration at each corresponding route and node will be used to influence the succeeding ants in making their decision in real-time. The node with the reduced concentration level $\tau_{l, n}$ will become less attractive to other ants. 
International Journal of Engineering Research and Technology. ISSN 0974-3154, Volume 13, Number 11 (2020), pp. 3072-3079

(C) International Research Publication House. https://dx.doi.org/10.37624/IJERT/13.11.2020.3072-3079

The local pheromone update is expressed as [15].

$$
\tau_{l . n}=(1-\xi) \tau_{l, n}^{t-1}+\xi \Gamma
$$

where $\tau_{l, n}^{t-1}$ is the previous pheromone value after evaporation. The local evaporation rate is $\xi$, and the initial pheromone is $\Gamma$. The step of updating the local pheromone enhances the probability of the ant to choose the node with higher pheromone concentration. The moment the maximum throughput with optimum power level is accomplished for each ant, this local solution will be chosen for a global pheromone update for that particular ant at that specific node. The ultimate optimal solution will be obtained after comparing all the available global updates on that node for all the ants. The global pheromone update is represented [15].

$$
\tau_{l, n}=(1-\rho) \tau_{l, n}^{t-1}+\rho R
$$

The global evaporation rate is $\rho$. $R$ is the highest throughput rate on a particular node with the chosen power level. There is a higher possibility for a more excellent solution on the node concerning the high deposition of pheromone. Moreover, when global pheromone updating is established, the initial iteration process is considered completed. The iterations will end when all ants keep visiting the same path with power levels on the subchannel that achieves the highest throughput. The algorithm is searching for the optimal distribution of the power to maximize the throughput under the system constraints known as minimum data rate and maximum transmit power.

Our proposed ACO algorithm will restrict the search by the ants in obtaining the optimal transmit power level, which yields maximum throughput within the maximum transmit power. The search will stop once the power level exceeds the maximum transmit power, and it will restart a new searching process again.

\section{IV.PERFORMANCE RESULT AND ANALYSIS}

The proposed algorithm has been simulated with the system parameters shown in Table 1. As reported in [15], the proposed ACO parameters are tuned based on trying different values recommended to attain the maximum of the fitness function. The initial pheromone is set to be $\Gamma=0.5$, where this pheromone concentration gives ants a chance to discover most of the available nodes. Furthermore, the set of the initial pheromone values change between 0.9 to 0.5 with fixing values of the other parameters as recommended by [15].

Hence, the design takes into account not to use low value of the initial pheromone, that can bias the ants to the first tour quickly. Also, the parameters for the regulation of the pheromone influence given as $\xi=\rho=0.1, \alpha=1, q_{0}=0, \beta=3$. The system performance was achieved by the ACO algorithm parameters with about 1000 maximum iterations.
Table 1. The setting of the System Parameters

\begin{tabular}{ll}
\hline Parameters & Setting \\
\hline Subchannel Bandwidth & $15 \mathrm{kHz}$ \\
Number of Subchannels & 128 \\
Maximum Transmit Power & $33 \mathrm{dBm}$ \\
Total Bandwidth & $5 \mathrm{MHz}$ \\
Cell Radius & $1000 \mathrm{~m}$ \\
Total Number of Users & $20 \mathrm{users}$ \\
Noise Power Spectral Density & $-174 \mathrm{dBm} / \mathrm{Hz}$ \\
Delay Extension & $5 \mathrm{us}$ \\
Maximum Doppler Shift & $30 \mathrm{~Hz}$ \\
Shadowing Standard Deviation & $8 \mathrm{~dB}$ \\
User Minimum Data Rate & $500 \mathrm{~b} / \mathrm{s}$ \\
Channel Estimation & Ideal \\
\hline
\end{tabular}

Fig. 4 shows the overall throughput (Mbps) versus the maximum transmit power $(\mathrm{dBm})$ for our proposed algorithm from $0 \mathrm{dBm}$ to $33 \mathrm{dBm}$. The obtained results are compared to the existing power allocation techniques such as the Fractional Transmit Power Allocation (FTPA) [16] [5], Equal Power Allocation (EPA) [8], and the Lagrange Multiplier (LM) [5] where their results reported are based on transmit power only between $10 \mathrm{dBm}$ to $20 \mathrm{dBm}$.

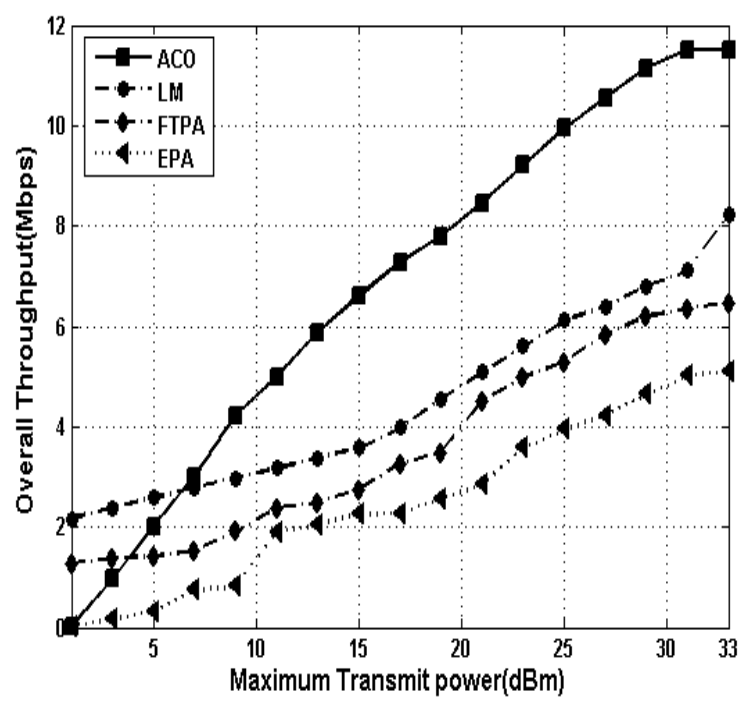

Fig. 4. Overall throughput versus maximum power allocation scheme for the downlink of NOMA system with 2 users

LM, FTPA, and EPA allocate power to the user multiplexed on the same subchannel based on the evaluated channel gain of the 
respective subchannel. This approach has added complexity to their algorithm [5].On the other hand, the proposed algorithm assigns the transmit power to the subchannel directly instead of assigning it based on the users' channel gains, which may vary from subchannel to another. The transmit power assignment based on repetitive fitness function evaluation and heuristic search between the power level nodes and subchannel nodes. This search has reduced the complexity and yet is able to yield better performance. Significant improvement in throughput is observed and tabulated in Table 2.

Table 2. Throughput performance comparison

\begin{tabular}{|l|c|c|c|}
\hline \multicolumn{3}{|c|}{ Throughput (Mbps) } \\
\hline \multirow{2}{*}{ Algorithms } & \multicolumn{2}{|c|}{ Transmit Power } & \multirow{2}{*}{$\begin{array}{c}\text { Performance } \\
\text { Gain }\end{array}$} \\
\cline { 2 - 3 } & $\mathbf{1 0} \mathbf{d B m}$ & $\mathbf{2 0 d B m}$ & \\
\hline ACO & 4.216 & 8.021 & - \\
\hline LM & 2.981 & 4.547 & $47.63 \%$ \\
\hline FTPA & 1.928 & 3.481 & $58.69 \%$ \\
\hline EPA & 0.813 & 2.574 & $68.43 \%$ \\
\hline
\end{tabular}

Table 2 demonstrates the performance gain of the proposed algorithm over the existing schemes based on transmit power range between $10 \mathrm{dBm}$ to $20 \mathrm{dBm}$. The ACO PA scheme outperforms the LM scheme [5] up to $47 \%$ of the overall throughput performance and the FTPA and EPA schemes more than 50\%.Furthermore, our proposed algorithm shows the upward trend of the throughput performance when the transmit power is extended from $20 \mathrm{dBm}$ to $33 \mathrm{dBm}$. The ACO algorithm demonstrates a considerable improvement of the system throughput over the other techniques. There is also a compensation for the distance impacts on the quality of the user's channel when suitable power levels are recommended for every multiplexed user with concerning of maximization of the fitness function. The proposed ACO PA is also able to operate in a different scenario for more than two users.

\section{IV.I MULTIUSER CASE}

Our proposed ACO algorithm is extended to more users. Here, we applied our proposed ACO algorithm in a NOMA system, as suggested in [16].For $U$ number of users in the NOMA system where the total bandwidth is divided into 8 sub-bands that are split into 6 subcarriers per sub-band [16]. Initially, the sub-band will be allocated equal powers while ACO is used to allocate the required powers for the subcarriers with maintaining $c_{1}$ constraints. To gain further insights into the effects of adopting more users in the system for the throughput performance of various power allocation techniques. Figure 5 illustrates the possible applications of ACO to improve the system throughput for a maximum number of users.

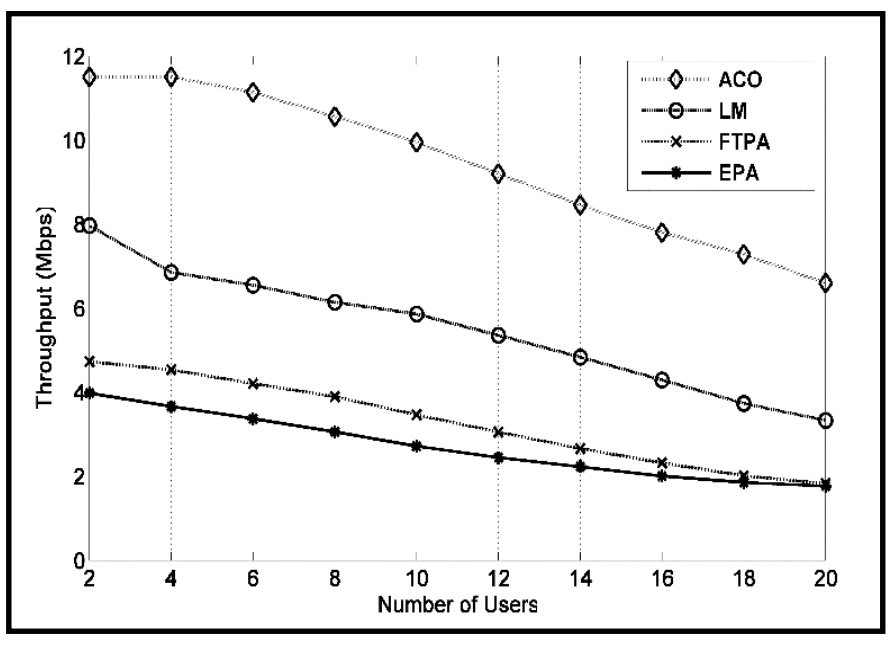

Fig.5. Throughput for maximum Number of Users

From Fig.5,it is observed that the performance of the throughput degrades as the number of users increases for all the techniques mainly due to the resource scarcity as more users need to share the same amount of subchannels. However, our proposed ACO technique is found to exhibit significant performance gain compared to the existing schemes. ACO technique offers more flexibility for the scheme of power levels allocation due to the quality of the search for solutions and the iterations that are able to enhance the system performance. The search solution becomes wider when more users are deployed, and the subsequence more power levels should be used in the search, which can ensure sufficient power levels for each pair of users multiplexed on the subchannel.

\section{CONCLUSION}

Ant Colony Optimization (ACO) is applied to optimize the transmit power distribution to maximize the overall throughput in a subchannel for the NOMA downlink system. The fitness function using the transmit power levels for the particular subchannel and power ratio for the multiplexed users has reduced the complexity of the power allocation problem. The search space in ACO gives a dynamic solution for power levels distribution based on subchannel since the power domain in the NOMA system pays higher priority to the power levels allocated for the users multiplexed on the same subchannel. Our proposed scheme's simulation results outperform FTPA, Lagrange Multiplier, and EPA in terms of throughput performance. 
International Journal of Engineering Research and Technology. ISSN 0974-3154, Volume 13, Number 11 (2020), pp. $3072-3079$

(C) International Research Publication House. https://dx.doi.org/10.37624/IJERT/13.11.2020.3072-3079

\section{REFERNCES}

[1] Dai, L., Wang, B., Yuan, Y., Han, S., Chih-Lin, I., \& Wang, Z. Non-orthogonal multiple access for 5G: solutions, challenges, opportunities, and future research trends. IEEE Communications Magazine, 53(9), 74-81,2015.

[2] Ding, Z., Liu, Y., Choi, J., Sun, Q., Elkashlan, M., Chih-Lin, I., \& Poor, H. V. (2017). Application of non-orthogonal multiple access in LTE and $5 \mathrm{G}$ networks. IEEE

Communications Magazine, 55(2), 185-191.

[3] Islam, S. R., Zeng, M., Dobre, O. A., \& Kwak, K. S. (2018). Resource allocation for downlink NOMA systems: Key techniques and open issues. IEEE Wireless Communications, 25(2), 4047.

[4] Parida, P., \& Das, S. S. (2014, December). Power allocation in OFDM based NOMA systems: A DC programming approach. In 2014 IEEE Globecom Workshops (GC Wkshps) (pp. 1026-1031). IEEE.

[5] Al-Abbasi, Z. Q., \& So, D. K. (2015, August). Power allocation for sum rate maximization in non-orthogonal multiple access system. In 2015 IEEE 26th Annual International Symposium on Personal, Indoor, and Mobile Radio Communications (PIMRC) (pp. 1649-1653). IEEE.

[6] Zhang, Z., Xia, Q., Yu, G., \& Liu, R. (2017, October). Power control, user scheduling and resource Allocation for Downlink NOMA Systems with Imperfect Channel State Information. In 2017 9th International Conference on Wireless Communications and Signal Processing (WCSP) (pp. 1-6). IEEE.

[7] Youssef, M. J., Farah, J., Nour, C. A., \& Douillard, C. (2017, July). Waterfilling-based resource allocation techniques in downlink Non-Orthogonal Multiple Access (NOMA) with Single-User MIMO. In 2017 IEEE Symposium on Computers and Communications (ISCC) (pp. 499-506). IEEE.

[8] Otao, N., Kishiyama, Y., \& Higuchi, K. (2012, August). Performance of non-orthogonal access with SIC in cellular downlink using proportional fair-based resource allocation. In 2012 international symposium on wireless communication systems (ISWCS) (pp. 476-480). IEEE.

[9] Saito, Y., Benjebbour, A., Kishiyama, Y., \& Nakamura, T. (2015, May). System-level performance of downlink non-orthogonal multiple access (NOMA) under various environments. In 2015 IEEE 81st Vehicular Technology Conference (VTC Spring) (pp. 1-5). IEEE.

[10] Sun, Y., Ng, D. W. K., Ding, Z., \& Schober, R. (2016, December). Optimal joint power and subcarrier allocation for MC-NOMA systems. In 2016 IEEE Global Communications Conference
(GLOBECOM) (pp. 1-6). IEEE.

[11] Gemici, Ö. F., Kara, F., Hokelek, I., Kurt, G. K., \& Çırpan, H. A. (2017, July). Resource allocation for NOMA downlink systems: Genetic algorithm approach. In 2017 40th International Conference on Telecommunications and Signal Processing (TSP) (pp. 114-118). IEEE.

[12] Dorigo, M., \& Gambardella, L. M. (1997). Ant colony system: a cooperative learning approach to the traveling salesman problem. IEEE Transactions on evolutionary computation, 1(1), 53-66.

[13] Huang, B., Li, J., \& Svensson, T. (2012, May). Joint scheduling for multi-service in coordinated multi-point OFDMA networks. In 2012 IEEE 75th Vehicular Technology Conference (VTC Spring) (pp. 1-5). IEEE.

[14] Yang, X. S. (2010). Engineering optimization: an introduction with metaheuristic applications. John Wiley \& Sons.

[15] Voss, S. (2006). Book Review: Morco Dorigo and Thomas Stützle: Ant colony optimization (2004) ISBN 0-262-04219-3, MIT Press, Cambridge. Mathematical Methods of Operations Research, 63(1), 191.

[16] Benjebbour, A., Saito, Y., Kishiyama, Y., Li, A., Harada, A., \& Nakamura, T. (2013, November). Concept and practical considerations of nonorthogonal multiple access (NOMA) for future radio access. In 2013 International Symposium on Intelligent Signal Processing and Communication Systems (pp. 770-774). IEEE. 